\title{
High Median Nerve Palsy Caused by Pseudoaneurysm After Brachial Catheterization: Two Case Reports
}

\author{
Kensuke Ochia, e, Goken Iwase ${ }^{\mathrm{b}}$, Sakiko Mizuno ${ }^{\mathrm{a}}$, Noboru Matsumurac ${ }^{\mathrm{c}}$, Takuji Iwamoto ${ }^{\mathrm{c}}$, \\ Itsuo Watanabe ${ }^{a}$, Hiraku Hotta ${ }^{\text {a }}$, Ukei Anazawa ${ }^{a}$, Kazuki Sato ${ }^{c}$, Shinichiro Takayama ${ }^{d}$
}

\begin{abstract}
We here present two very rare cases of high median nerve palsy caused by pseudoaneurysm after brachial catheterization. A 71-yearold woman developed high median nerve palsy 2 weeks after brachial catheterization. She underwent pseudoaneurysm resection together with neurolysis 4 months after the onset of palsy. Surgical findings suggested that her palsy was caused by both severe compression by pseudoaneurysm and adhesion following hematoma after catheterization. Five months after the surgery, she only had slight sensory disturbance. A 48-year-old lady developed high median nerve palsy 1 week after the catheterization. Simple pseudoaneurysm resection was performed 2 weeks after the catheterization. Neurolysis was not performed. Seven months after the surgery, she still had severe sensory disturbance. Our cases suggested importance of secured astriction after catheterization, and recommended surgical procedure for this condition is combination of pseudoaneurysm resection and neurolysis by hand surgeons.
\end{abstract}

Keywords: Brachial artery; Median nerve palsy; Brachial catheterization; Pseudoaneurysm resection; Neurolysis; Hand surgeon

\section{Introduction}

According to the aging of our population, incidence of ischemic heart disease is increasing these days. Brachial catheterization is one of the most common treatments for ischemic heart

Manuscript accepted for publication December 06, 2016

aDepartment of Orthopaedic Surgery, Tokyo Dental College, Ichikawa General Hospital, 5-11-13 Sugano, Ichikawa, Chiba 272-8513, Japan

bepartment of Orthopaedic Surgery, Saiseikai Mito Hospital, Mito, Ibaragi, Japan

${ }^{\mathrm{c}}$ Department of Orthopaedic Surgery, Keio University School of Medicine, Shinjuku, Tokyo, Japan

${ }^{\mathrm{d} D e p a r t m e n t}$ of Orthopaedic Surgery, National Center for Child Health and Development, Setagaya, Tokyo, Japan

eCorresponding Author: Kensuke Ochi, Department of Orthopaedic Surgery, Tokyo Dental College, Ichikawa General Hospital, 5-11-13 Sugano, Ichikawa, Chiba 272-8513, Japan. Email: ochi@1998.jukuin.keio.ac.jp

doi: https://doi.org/10.14740/jmc2715w disease, and although very few, median nerve palsy caused by hematoma following brachial catheterization is a well-known complication after this treatment [1-4]. However, there are few cases of high median nerve palsy caused by pseudoaneurysm after brachial catheterization in English literature to the best of our knowledge $[5,6]$. The purpose of this report was to present two cases of high median nerve palsy caused by pseudoaneurysm after brachial catheterization, and also to propose how to prevent and treat it.

\section{Case Reports}

\section{Case 1}

A 71-year-old woman visited our clinic with a chief complaint of difficulty in flexing her thumb and index finger. She first recognized difficulty in flexing her fingers 2 weeks after brachial catheterization for treating myocardial infarction. She had a pulsative, palpable soft tissue mass (about $3 \mathrm{~cm}$ in its diameter) on the flexor side of her elbow about $2 \mathrm{~cm}$ proximal to her medial epicondyle line (Fig. 1a), and had tender and a Tinel-like sign on the mass. Angiography confirmed that her mass was pseudoaneurysm at brachial artery (Fig. 1b). Ultrasonography and magnetic resonance angiography were not performed since these could not be done at the time of her treatment (in the year of 2001). Both her flexor pollicis longus (FPL) and flexor digitorium profundus of the index finger (FDP1) showed manual muscle power of grade 2 (British MRC grading), and had abductor pollicis brevis (APB) muscle atrophy. She also had sensory disturbance in her median nerve region, and electrophysiological examination confirmed her high median nerve palsy. Since she refused early surgical treatment, she was conservatively treated for 4 months after the onset of her palsy. However, she showed no sign of recovery. Thus, neurolysis together with pseudoaneurysm resection was performed. Surgical findings confirmed that her median nerve was severely compressed by pseudoaneurysm (Fig. 1c), with pseudoneuroma on its proximal side (Fig. 1d). Her median nerve was also severely adhered to surrounding tissue, suggesting that not only pseudoaneurysm but also hematoma after catheterization was the cause of her palsy. Five months after the surgery, both her FPL and FDP1 had muscle powers of grade 5 and her sensory disturbance showed significant 

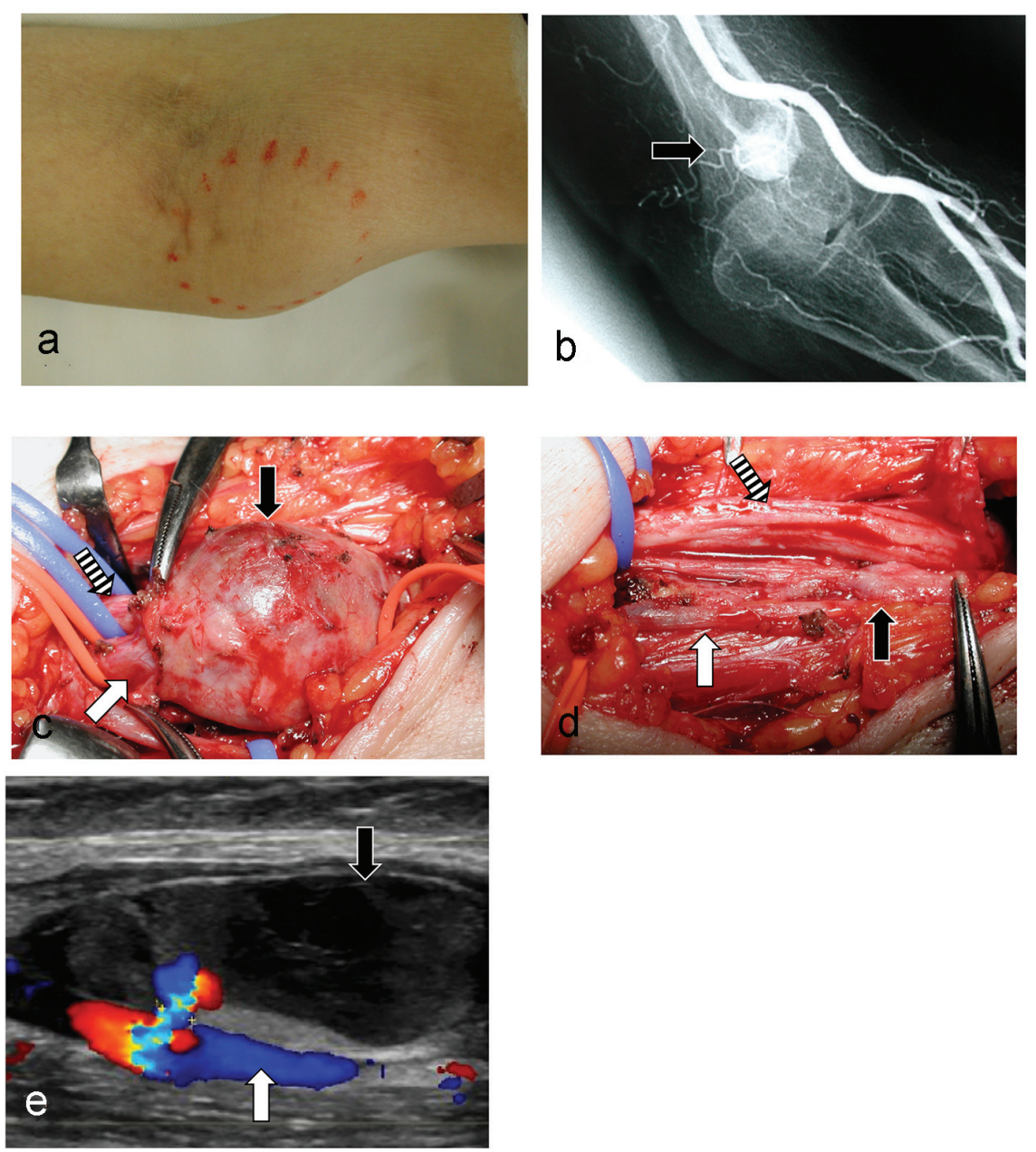

Figure 1. Demonstrative figures of case 1 and case 2. (a) Case 1. A soft tissue tumor with a Tinel-like sign seen on the flexor side of her elbow at the level of her medial epicondyle (circled). (b) Case 1. Angiography confirmed that her soft tissue tumor was pseudoaneurysm at brachial artery (black arrow). (c) Case 1. Surgical findings before pseudoaneurysm resection. Black arrow: pseudoaneurysm, Shadowed arrow: median nerve, White arrow: brachial artery (d) Case 1. Surgical findings after pseudoaneurysm resection and neurolysis. Black arrow: pseudoneuroma of median nerve seen after resection of pseudoaneurysm, Shadowed arrow: median nerve, White arrow: brachial artery. (e) Case 2. Doppler ultrasonography. Black arrow: pseudoaneurysm, White arrow: brachial artery.

recovery.

\section{Case 2}

A 48-year-old lady visited our clinic with a complication of difficulty in flexing her thumb and index finger. She lost consciousness because of cardiac tamponade, and underwent brachial catheterization as one of her treatments. She restored consciousness on the day of the catheterization, and first recognized difficulty in flexing her fingers 1 week after the catheterization. She had marked subcutaneous hematoma caused by the catheterization on the ulnar side of her distal arm, suggesting that she was in mortal danger from the cardiac tamponade and astriction after catheterization might be insufficient. Both Tinel-like sign and tenderness were seen on the hematoma. Apparent soft tissue mass or pulsation was not palpable be- cause of skin stiffness caused by the severe hematoma. Muscle power of her FPL was grade 1, and that of her FDP1 was grade 2 . She had sensory disturbance in her median nerve region, and was clinically diagnosed as high median nerve palsy. Ultrasonography confirmed presence of about a size of $3.5 \mathrm{~cm}$ pseudoaneurysm at brachial artery approximately $3 \mathrm{~cm}$ proximal to her medial epicondyle line (Fig. 1e). Simple resection of pseudoaneurysm was performed by vascular surgeons 2 weeks after the catheterization. Neurolysis was not performed, since hand surgeon did not attend to this surgery. Seven months after the surgery, both her FPL and FDP1 were grade 5, but she still had severe sensory disturbance in her median nerve region.

\section{Discussion}

Here we report two cases of high median nerve palsy caused 
by pseudoaneurysm after brachial catheterization. High median nerve palsy caused by hematoma after brachial catheterization is occasionally reported; however, there are only two well-described cases in literature similar to ours as far as we know. Although condition like ours seems to be extremely rare at this point of time, this condition should increase in the future according to the increase of ischemic heart disease caused by aging of our population. Hence, it is very important to be aware of this condition, and perform accurate prevention.

Although it is impossible to completely prevent pseudoaneurysm, secured astriction seems to be the most reliable procedure to prevent it. Previous reports have shown that pseudoaneurysm develops from vascular wall damaged by catheterization, and secure astriction could prevent development of pseudoaneurysm [7]. Secured astriction can also reduce subcutaneous hemorrhage after catheterization, which is effective for decreasing median nerve palsy caused by hematoma following catheterization [7]. Although a simple and classic method, we believe having cardiovascular physicians to thoroughly re-recognize importance of secured astriction is the most important prevention for this condition. Another prevention is to stop using brachial catheterization and use radial artery in the wrist as the entry point for catheterization. But this method still has risks of complications including pseudoaneurysm and damage of the radial sensory nerve [8].

Treatment of a palsy caused by pseudoaneurysm is still controversial. However, based on our two cases and other reported cases, we believe that surgery should be considered in cases that do not show spontaneous recovery within $3-6$ months, and neurolysis together with resection of pseudoaneurysm should be recommended for following reasons: 1) more than half of the pseudoaneurysm enlarges without disappearing spontaneously [9]; 2) although few, all the cases persisted functional impairment even though they were surgically treated; 3 ) the median nerve was severely adhered to surrounding tissue and was compressed because of hematoma after catheterization; and 4) our case 1 (neurolysis together with pseudoaneurysm resection) showed better functional recovery compared to our case 2 (simple resection of pseudoaneurysm).

We reported two cases of high median nerve palsy caused by pseudoaneurysm after brachial catheterization. Our cases together with literature suggested that secured astriction may be the best prevention for palsies caused by pseudoaneurysm after catheterization. Surgical treatment of neurolysis together with pseudoaneurysm resection should be recommended if such cases do not show spontaneous recovery within several months.

\section{Acknowledgments}

We thank all the members of the Department of Orthopedic
Surgery, Tokyo Dental College Ichikawa General Hospital, and members of the Department of Orthopedic Surgery, Keio University School of Medicine for their support.

\section{Grant Support}

This work was supported in part by grants-in-aid for scientific research from the Japan Society for the Promotion of Science (No. 15K10421) to KO.

\section{Conflicts of Interest}

None.

\section{References}

1. Littler WA. Median nerve palsy - a complication of brachial artery cannulation. Postgrad Med J. 1976;52(Suppl 7):110-113

2. Winer JB, Harrison MJ. Iatrogenic nerve injuries. Postgrad Med J. 1982;58(677):142-145.

3. Kennedy AM, Grocott M, Schwartz MS, Modarres H, Scott M, Schon F. Median nerve injury: an underrecognised complication of brachial artery cardiac catheterisation? J Neurol Neurosurg Psychiatry. 1997;63(4):542546.

4. Ikeda K, Osamura N. Median nerve palsy: a complication of brachial artery cardiac catheterization. Hand Surg. 2011;16(3):343-345.

5. Yip KM, Yurianto H, Lin J. False aneurysm with median nerve palsy after iatrogenic brachial artery puncture. Postgrad Med J. 1997;73(855):43-44.

6. Fukuda K, Nishiura Y, Suzuki S, Ochiai N. High median nerve palsy after brachial artery catheterization: a case report. Peripheral Nerve. 2003;14(1):66-69.

7. Applegate RJ, Sacrinty MT, Kutcher MA, Baki TT, Gandhi SK, Santos RM, Little WC. Propensity score analysis of vascular complications after diagnostic cardiac catheterization and percutaneous coronary intervention 19982003. Catheter Cardiovasc Interv. 2006;67(4):556-562.

8. Nagai S, Abe S, Sato T, Hozawa K, Yuki K, Hanashima $\mathrm{K}$, Tomoike H. Ultrasonic assessment of vascular complications in coronary angiography and angioplasty after transradial approach. Am J Cardiol. 1999;83(2):180-186.

9. Hirano Y, Ikuta S, Uehara H, Nakamura H, Taniguchi M, Kimura A, Hayashi T, et al. [Diagnosis of vascular complications at the puncture site after cardiac catheterization]. J Cardiol. 2004;43(6):259-265. 